\title{
Feasibility of progressive sit-to-stand training among older hospitalized patients
}

Mette Merete Pedersen, Janne Petersen, Jonathan F Bean, Lars LD Damkjaer, Helle Gybel Juul-Larsen, Ove Andersen, Nina Beyer, Thomas Bandholm

Background. In older patients hospitalization is associated with a decline in functional performance and loss of muscle strength. Loss of muscle strength and functional performance can be prevented by systematic strength training but details are lacking regarding the optimal exercise program and dose for older patients. Therefore, our aim was to test the feasibility of a progression model for loaded sit-to-stand training among older hospitalized patients. Methods. This is a prospective cohort study conducted as a feasibility study prior to a full-scale trial. We included twenty-four older patients ( $\geq 65 \mathrm{yrs}$ ) acutely admitted from their own home to the medical services of the hospital. We developed an 8-level progression model for loaded sit-to-stands, which we named STAND. We used STAND as a model to describe how to perform the sit-to-stand exercise as a strength training exercise aimed at reaching a relative load of 8-12 repetitions maximum (RM) for 8-12 repetitions. Weight could be added by the use of a weight vest when needed. The ability of the patients to reach the intended relative load (8-12 RM), while performing sit-to-stands following the STAND model, was tested once during hospitalization and once following discharge in their own homes. A structured interview including assessment of possible modifiers (cognitive status by the Short Orientation Memory test and mobility by the de Morton Mobility Index) was administered both on admission to the hospital and in the home setting. The STAND model was considered feasible if: 1) $75 \%$ of the assessed patients could perform the exercise at a given level of the model reaching 8-12 repetitions at a relative load of 8-12 RM for one set of exercise in the hospital and two sets of exercise at home; 2) no ceiling or floor effect was seen; 3) no indication of adverse events were observed. The outcomes assessed were: level of STAND attained, the number of sets performed, perceived exertion (the Borg scale), and pain (the Verbal Ranking Scale).

Results. Twenty-four patients consented to participate. Twenty-three of the patients were tested in the hospital and 19 patients were also tested in their home. All three criteria for feasibility were met: 1 ) In the hospital, $83 \%$ could perform the exercise at a given level of STAND, reaching 8-12 repetitions at 8-12 RM for one set, and $79 \%$ could do so for two sets in the home setting; 2) for all assessed patients, a possibility of progression or regression was possible - no ceiling or floor effect was observed; 3 ) no indication of adverse events 
(pain) was observed. Also, those that scored higher on the de Morton Mobility Index performed the exercise at higher levels of STAND, whereas performance was independent of cognitive status. Conclusions. We found a simple progression model for loaded sit-tostands (STAND) feasible in acutely admitted older medical patients ( $\geq 65 \mathrm{yrs}$ ), based on our pre-specified criteria for feasibility. 


\section{Author cover page}

2 Title

3 Feasibility of progressive sit-to-stand training among older hospitalized patients 4

5 Mette Merete Pedersen ${ }^{1,2}$, Janne Petersen ${ }^{1,3}$, Jonathan F. Bean ${ }^{4,5}$, Lars LD Damkjær ${ }^{6}$, Helle

6 Gybel Juhl-Larsen ${ }^{1,2}$, Ove Andersen ${ }^{1}$, Nina Beyer ${ }^{7}$, Thomas Bandholm ${ }^{1,2,8}$

8 1) Optimized Senior Patient Program (Optimed), Clinical Research Centre, Hvidovre Hospital,

9 University of Copenhagen, Hvidovre, Denmark

10 2) Physical Medicine \& Rehabilitation Research-Copenhagen (PMR-C); Department of Physical

11 and Occupational Therapy, Hvidovre Hospital, University of Copenhagen, Hvidovre, Denmark

12 3) Section of Biostatistics, Department of Public Health, University of Copenhagen, Denmark

13 4) New England GRECC, VA Boston Healthcare System, Boston, MA, USA

14 5) Department of Physical Medicine and Rehabilitation, Harvard Medical School, Boston, MA, 15 USA

16 6) Department of Rehabilitation, Copenhagen Municipality Health Administration, Copenhagen,

17 Denmark

18 7) Institute of Sports Medicine \& Musculoskeletal Rehabilitation Research Unit, Bispebjerg

19 Hospital, Bispebjerg, Denmark

20 8) Department of Orthopaedic Surgery, Hvidovre Hospital, University of Copenhagen, Hvidovre,

21 Denmark

23 Corresponding author:

24 Mette Merete Pedersen, Clinical Research Centre (056), Hvidovre Hospital, University of

25 Copenhagen, DK-2650 Hvidovre, Denmark. Email: mette.merete.pedersen@regionh.dk; phone:

26 +453862 1590; Fax: +4538623797. 
27 Abstract

28

29 Background. In older patients hospitalization is associated with a decline in functional

30 performance and loss of muscle strength. Loss of muscle strength and functional performance

31 can be prevented by systematic strength training but details are lacking regarding the optimal exercise program and dose for older patients. Therefore, our aim was to test the feasibility of a progression model for loaded sit-to-stand training among older hospitalized patients.

Methods. This is a prospective cohort study conducted as a feasibility study prior to a full-scale trial. We included twenty-four older patients ( $\geq 65 \mathrm{yrs})$ acutely admitted from their own home to the medical services of the hospital. We developed an 8-level progression model for loaded sitto-stands, which we named STAND. We used STAND as a model to describe how to perform the sit-to-stand exercise as a strength training exercise aimed at reaching a relative load of 8-12 repetitions maximum (RM) for 8-12 repetitions. Weight could be added by the use of a weight vest when needed. The ability of the patients to reach the intended relative load (8-12 RM), while performing sit-to-stands following the STAND model, was tested once during hospitalization and once following discharge in their own homes. A structured interview including assessment of possible modifiers (cognitive status by the Short Orientation Memory test and mobility by the de Morton Mobility Index) was administered both on admission to the hospital and in the home setting. The STAND model was considered feasible if: 1) $75 \%$ of the assessed patients could perform the exercise at a given level of the model reaching 8-12 repetitions at a relative load of 8-12 RM for one set of exercise in the hospital and two sets of exercise at home; 2) no ceiling or floor effect was seen; 3) no indication of adverse events were observed. The outcomes assessed were: level of STAND attained, the number of sets performed, perceived exertion (the Borg scale), and pain (the Verbal Ranking Scale). Results. Twenty-four patients consented to participate. Twenty-three of the patients were tested in the hospital and 19 patients were also tested in their home. All three criteria for feasibility were met: 1) In the hospital, 83\% could perform the exercise at a given level of STAND, reaching 8-12 repetitions at 8-12 RM for one set, and 79\% could do so for two sets in the home setting; 2) for all assessed patients, a possibility of progression or regression was possible - no ceiling or floor effect was observed; 3 ) no indication of adverse events (pain) was observed. Also, those that scored higher on the de Morton Mobility Index performed the exercise at higher levels of STAND, whereas performance was independent of cognitive status. 
59 Conclusions. We found a simple progression model for loaded sit-to-stands (STAND) feasible

60 in acutely admitted older medical patients ( $\geq 65 \mathrm{yrs}$ ), based on our pre-specified criteria for

61 feasibility.

62

63 Introduction

64 In older hospitalized medical patients, self-reported decline in functional skills is common before

65

66

67

68

69 and during hospitalization (Covinsky et al., 2003; Brown, Friedkin \& Inouye, 2004; Boyd et al., 2008; Mudge, O’Rourke \& Denaro, 2010; Oakland \& Farber, 2014; Zisberg et al., 2015) and associated with low in-hospital mobility (Brown, Friedkin \& Inouye, 2004; Zisberg et al., 2015); $30-35 \%$ experience a decline in the ability to perform Activities of Daily Living (ADL) from admission to discharge (Covinsky et al., 2003; Boyd et al., 2008) and barely one third of these patients return to their preadmission level within the first year after discharge (Boyd et al., 2008).

In healthy older adults, even a few days of experimental immobilization or periods of bed rest can reduce muscle strength and functional performance (Kortebein et al., 2007; Hvid et al., 2010, 2014; Coker et al., 2014). Also, older adults are more sensitive to bed rest inactivity and have an impaired ability to fully recover compared to younger adults (Kortebein, 2009; Hvid et al., 2010, 2014). Lower activity levels are common among hospitalized older adults (Pedersen et al., 2012; Villumsen et al., 2014), and are linked to a decline in functional performance and associated with new institutionalization and death (Brown, Friedkin \& Inouye, 2004; Zisberg et al., 2015). Moreover, hospitalization is associated with a subsequent loss of muscle strength (Alley et al., 2010), putting hospitalized older adults at a higher risk of losing independence as a consequence of their hospitalization. Maintaining independence is considered the most important health outcome by many older adults (Fried et al., 2011). Therefore, preventing inactivity and loss of muscle strength and functional performance during hospitalization may well be a way of preventing loss of independence.

According to recent systematic reviews, loss of muscle strength and functional performance can be prevented by systematic strength training in both healthy and ill older adults (de Morton, Keating \& Jeffs, 1996; Kraemer \& Ratamess, 2004; Liu \& Latham, 2009; Koopman \& van Loon, 2009; Stewart, Saunders \& Greig, 2014). Also, strength training initiated during 
90 hospitalization can prevent decline in strength and functional performance associated with

91 hospitalization (Sullivan et al., 2001; Suetta et al., 2007). In addition, beneficial effects of

92 strength training on functional performance are reported among newly discharged older adults

93 and among frail community-dwelling older adults (Chandler et al., 1998; Courtney et al., 2012).

94 In general, exercise programmes for older hospitalized or community-dwelling adults consist of 95 a range of exercises (Chandler et al., 1998; Siebens et al., 2000; Alexander et al., 2001; Bean et

96 al., 2004; Brown et al., 2006; Nolan \& Thomas, 2008; Courtney et al., 2012; Tibaek et al., 2013; 97 Abrahin et al., 2014). Few studies have examined the effect of a cross-continuum program 98 initiated during hospitalization and continued after discharge (Siebens et al., 2000; Brown et al., 99 2006). Moreover, these previous studies have experienced problems with compliance (Siebens et 100 al., 2000; Brown et al., 2006) necessitating the importance of ongoing supervision from trained 101 staff even within the home setting (Siebens et al., 2000; Brown et al., 2006; Wall, Dirks \& van 102 Loon, 2013). Additionally, details are lacking regarding the optimal nature and dose of exercise 103 (De Morton, Keating \& Jeffs, 2009a; Liu \& Latham, 2009; Steib, Schoene \& Pfeifer, 2010). It 104 appears, though, that higher intensities are superior to lower intensities in older adults (Nicola \& 105 Catherine, 2011; Raymond et al., 2013; White et al., 2015).

106 The ideal exercise program for a hospitalized patient should be feasible to perform within a busy 107 care setting. It should be relatively simple requiring minimal equipment and also address the 108 impairments (poor limb strength) and functional deficits (poor mobility skills) common to 109 hospitalized patients (Bodilsen et al., 2013; De Buyser et al., 2014). Therefore, we focused upon 110 repeated sit-to-stand exercises, since it meets all of these criteria. Our aim was to test the 111 feasibility of a model for progressive sit-to-stand training among older hospitalized patients.

112 Specifically, we wanted to investigate if the progression model would enable the patients to 113 reach a strength training intensity of 8-12 repetitions maximum (RM) for 8-12 repetitions during 114 hospitalization and shortly following discharge, with no indications of ceiling or floor effects for 115 loading, no indications of adverse events and with acceptable exercise adherence.

\section{Methods}

118 Study design

119 The study is a prospective cohort study conducted as a feasibility study (Bowen et al., 2009;

120 Arain et al., 2010; Abbott, 2014) to indicate the feasibility of a progression model for loaded sit121 to-stands when used as a simple strength training exercise. The study was performed from 
122 December 2012 to July 2013. Participants were included to test their ability to perform the

123 progressive sit-to-stand exercise once in the hospital and once in their own homes within the first

124 two weeks following discharge. Inclusion took place at Hvidovre Hospital, University of

125 Copenhagen, Hvidovre, Denmark. The feasibility study was performed prior to a full-scale

126 randomized controlled trial (ClinicalTrials.gov-identifier: NCT01964482). All participants were

127 informed about the study verbally and in writing before providing written informed consent. The

128 local ethics committee approved the study (H-2-2012-115). The reporting of the study follows

129 the Strengthening the Reporting of Observational Studies in Epidemiology (STROBE) guidelines

130 for cohort studies (von Elm et al., 2014), and the description of the intervention follows the

131 Template for Intervention Description and Replication (TIDieR) checklist (Hoffmann et al.,

132 2014). When we designed the present study, endorsement of registration of all trials was not as

133 prevalent as today, which is why it was not registered. All criteria related to feasibility, however,

134 were pre-specified.

135

136 Subjects

137 Older medical patients ( $\geq 65 \mathrm{yrs}$ ) acutely admitted from their own home to the medical services

138 of the hospital, via the emergency department, were included by random sampling. The

139 exclusion criteria were: 1) inability to rise from a chair with help; 2) inability to cooperate in

140 measurements; 3) inability to give informed consent to participate; 4) diagnosis of Chronic

141 Obstructive Pulmonary Disease (COPD) and participation in a COPD rehabilitation program; 5)

142 terminal illness or being in cancer treatment; 6) inability to speak or understand Danish; 7)

143 isolation-room stay; 8) transferral to the intensive care unit; 9) an expected hospitalization of one

144 day or less.

\section{Procedures}

147 All assessments were performed by two skilled physiotherapists - one with 15 years of

148 experience (the primary investigator, MMP), and one with two years of experience (HGJ). The

149 same physiotherapist performed all assessments for a given patient. Before initiation of the

150 study, HGJ was trained in all assessments and the progression model and assisted MMP in

151 assessing the first two patients to ensure standardization. 


\section{Descriptive data}

154 Medical records were extracted for demographic data, co-morbidities, length of hospital stay, 155 admission diagnosis, and discharge destination. The patients underwent a structured baseline 156 interview within the initial 48 hours of the hospital stay, to collect information about marital 157 status, residence before hospitalization, recent weight loss, basic mobility, functional 158 independence, physical activity level 2 weeks prior to admission, health status, nutritional status, 159 cognitive status, and mobility: The Cumulated Ambulation Score (CAS) was used as an 160 objective measure of basic mobility. It quantifies the patients' independence in three basic 161 activities: getting in and out of bed, sit-to-stand from a chair, and walking (Foss, Kristensen \& 162 Kehlet, 2006); The New Mobility Score (NMS) was used to assess functional independence in 163 retrospect 2 weeks before admission and in retrospect over the day of admission, respectively 164 (Parker \& Palmer, 1993); the level of self-reported physical activity was assessed by a questionnaire modified by Schnohr (Saltin \& Grimby, 1968; Schnohr, Scharling \& Jensen, 2003) categorizing physical activity of the patient in level 1: low physical activity, level 2: moderate physical activity, and levels 3+4: high physical activity; The EQ-VAS of the EQ-5D was used to assess health status (Rabin \& de Charro, 2001); and Nutritional Risk Screening (NRS) was used to screen for nutritional risk (Kondrup, 2003). In addition, two possible modifiers were assessed both on admission and in the patients' own homes: 1) the de Morton Mobility Index (DEMMI) (score 0-100) to quantify the patient's mobility level before performing the exercise (de Morton,

172 Davidson \& Keating, 2008). A level of $<62$ is below normative values for community-dwelling 173 older adults and thus considered to reflect limited mobility (Macri et al., 2012); 2) The Short

174 Orientation-Memory-Concentration test (OMC) to assess cognitive status (Katzman et al., 1983).

175 A score of 0 reflects the worst cognitive status and a score of 28 reflects the best cognitive status. A score $\leq 22$ was considered to reflect impaired cognition (Wade \& Vergis, 1999).

179 We developed a progression model for loaded sit-to-stands as a strength training exercise and 180 named the model STAND (Figure 1). STAND was intended to be suitable for older medical patients in the hospital and in their own homes and to ensure training to muscular fatigue in both

182 settings. While developing STAND several meetings were held with physiotherapists from the 183 municipality of Copenhagen to include their ideas on the contents of the different levels of the 184 model. Within 48 hours of admission, the patients were contacted at the ward by one of the two 
185 physical therapists to test their ability to perform a sit-to-stand strength training exercise for the 186 lower extremities (acute-phase feasibility). On day one or two after discharge from the hospital 187 the patients were contacted again by telephone to arrange a re-test of the ability to perform the 188 strength training exercise in their own homes (stable-phase feasibility). The difficulty of the 189 exercise was predefined by STAND ensuring exercise to muscular fatigue in every exercise set 190 (Figure 1). The easiest level of STAND (level 1) was seated knee-extensions with or without a 191 weight-cuff, which simulates some of the muscle actions required to go from sit- to- stand.

192 Weight cuffs of $0.5 \mathrm{~kg}, 1 \mathrm{~kg}, 1.5 \mathrm{~kg}, 2 \mathrm{~kg}, 3 \mathrm{~kg}, 4 \mathrm{~kg}$ and $5 \mathrm{~kg}$ were used. The most difficult level 193 (level 8) was squat on one leg with added extra weight in the form of a weight vest (Titan Box, $19430 \mathrm{~kg}$ ). The vest had 30 pockets, 15 on the front and 15 on the back, each of which could contain 195 a $1 \mathrm{~kg}$ weight - the maximal load of the vest being $30 \mathrm{~kg}$.

196 The patient was seated on a standard chair with armrests, and a seat height of approximately 45 $197 \mathrm{~cm}$. As a warm-up exercise, the patient was asked to perform five unloaded knee extensions for each limb. The starting point in STAND was level 5 (Figure 1): sit-to-stand with arms crossed over the chest. From at seated position, the patient was asked to rise to a fully extended position and to sit down in a constant pace. The patient was verbally encouraged to perform as many repetitions as possible maintaining the same pace to ensure training to muscular fatigue (Tan, 1999). All exercises were performed at a moderate velocity with both the concentric (raising) and the eccentric (lowering) component being performed over two seconds, separated by a onesecond isometric pause after the concentric and eccentric phases, respectively (Kraemer \& Ratamess, 2004). Both sessions (in-hospital and at home) aimed at three sets of 8-12 repetitions maximum (henceforth: 8-12 RM) corresponding to training at 60-70\% of 1 RM (Tan, 1999; Kraemer et al., 2002; Kraemer \& Ratamess, 2004). In each set, the aim was to reach fatigue at 812 RM (Kraemer \& Ratamess, 2004), and the correct level of STAND was chosen accordingly (Figure 1). A two-minute pause was held between sets (Kraemer \& Ratamess, 2004). In order to ensure that an appropriate training load was achieved, a given level of training was accepted if

211 the patient could perform six non-compensatory repetitions and needed extra support performing

212 the last repetitions (e.g. minimal use of armrests) as long as a proper technique could be

213 maintained. Moreover, increased speed in the concentric phase was allowed in the last two

214 repetitions to optimize limb power output, as leg power has been shown to be associated with 215 physical performance in mobility-limited older adults (Bassey et al., 1992; Bean et al., 2002). 
216 The same skilled physical therapist supervised all exercise sessions and assessed the level of

217 each patient throughout the sets. The duration of each exercise session was 10-15 minutes.

218

219 Outcomes measures

220 Criteria for feasibility

221 STAND was considered feasible if three criteria were fulfilled: 1) $75 \%$ of the assessed acute222 phase patients and stable-phase patients, respectively, could perform the exercise at a given level 223 of the model without session failure. In the hospital, a session failure was defined as inability to 224 perform at least one set of 8-12 RM, and at home a session failure was defined as inability to 225 perform at least two sets of 8-12 RM. One to three sets are recommended for improving 226

muscular strength in older adults (Kraemer \& Ratamess, 2004) and both one set and multiple sets have been shown to be efficient in improving physical performance and muscle strength in older women (Abrahin et al., 2014). Thus, a smaller training volume was accepted in the acute-phase. All causes of session failure were recorded; 2) no clustering of patients at the lowest level (level 1) or the highest level (level 8) was seen - no ceiling or floor effect; 3 ) no indication of adverse events were observed, e.g. no persistent increase in pain.

\section{Training level and-load}

For each set in the two sessions (in-hospital and at home), the level in STAND, the extra load added $(\mathrm{kg})$, and the number of repetitions were noted.

\section{The Borg Scale}

The Borg Scale was administered immediately after each set of the exercise as a measure of perceived exertion (Borg, 1970). In healthy older adults, a Borg score of 14-16 has been shown to correspond to $70-90 \%$ of 1 RM (Row, Knutzen \& Skogsberg, 2012) and the Borg score was used as an indicator of whether the perceived effort corresponded with the RM level.

\section{The Verbal Ranking Scale (VRS)}

Before and after assessment of the DEMMI and before, during, and 10 minutes after the exercise, the patients were asked if they felt pain and wherefrom by the use of the VRS (Melzack, 1975). 
246 The absence of pain was not a feasibility criterium, but information on pain was collected to gain

247 knowledge about potential adverse events.

248

249 Statistical analysis

250 No formal sample size calculation was performed due to the descriptive character of the study

251 and as no efficacy testing was to be performed (Arain et al., 2010; Abbott, 2014). However, a

252 sample size of 24 was decided to be sufficient to obtain a proper variability in the functional

253 level of the patients and thereby be able to evaluate the feasibility of the model in older medical

254 patients. The feasibility results are presented as descriptive data given as means with standard

255 deviations, medians with inter-quartile ranges or percentages, depending on variable type. To

256 evaluate if the level of STAND depended on mobility and cognition, linear regression analyses

257 were used to regress the level of STAND on DEMMI and OMC, respectively. Change in

258 performance measures from admission to at home was tested using Wilcoxon Signed Rank test

259 and the paired t-test depending on variable type. All data were double entered in the programme

260 'Epidata Software' (version 3.1) and all data management and analyses were performed using the

261 SAS version 9.3.

262

263 Results

264 Patient characteristics

265 A total of 248 patients were assessed for eligibility and fulfilled the inclusion criteria. Of these

266200 were excluded based on our exclusion criteria: six were unable to rise from a chair with

267 help; 65 were not able to participate (e.g., due to dementia or confusion); one was participating

268 in a COPD rehabilitation program; 15 were in cancer treatment or terminally ill; four were

269 unable to speak or understand Danish; three were transferred to an isolation room; and 106 were

270 discharged within the first 24 hours (Figure 2). Forty-eight were asked to participate in the study.

271 Of these, 24 patients consented to participate in interviews and tests and 24 declined to

272 participate. The patients were included over a period of 13 weeks with an average inclusion of

2731.8 patients per week. One patient dropped out during the initial examination, leaving 23 patients

274 to be tested at the hospital. Two patients did not want the following home visit, one patient

275 declined to participate in testing at home, and one patient was unable to participate due to

276 worsening of disease, leaving 19 patients to be tested at home. Thus, a total of $20.8 \%$ dropped 
277 out of the study. Patient characteristics are presented in Table 1. No patients changed in CAS

278 from admission to follow-up. Also, no significant change was seen in NMS and DEMMI

279 whereas self-rated health improved significantly (Tabel 2).

280

281 Feasibility

282 Sets and loading

283 At the hospital, 20 of the 23 patients (83\%) were able to perform at least one set of 8-12 RM at a 284 given level of STAND - the remaining three patients stopped after 6-7 repetitions; one due to 285 dyspnea, one due to muscular fatigue, and one due to back pain that was present before 286 performing the exercise. All three patients were subsequently able to perform several sets of 8-12 287 RM in their own home.

288

At home, 15 of the 19 patients (79\%) were able to perform two sets of 8-12 RM, and 8 of these were able to perform three sets of 8-12 RM. Reasons for not attaining the goal of two sets of 812 RM were: one patient could perform seven repetitions in set one and 10 repetitions in set two; one patient stopped after one set due to knee pain - this pain did not persist after ending the exercise; one patient wanted to stop after one set due to a sensation of muscular fatigue during the first set; one patient wanted to stop in set two due to a sensation of muscular fatigue.

The 20 patients completing one set at the hospital were distributed in STAND as follows: two seated knee extensions, two sit-to-stand using the arm rests when standing and sitting down, two sit-to-stand using the arm rests when sitting down, six sit-to-stand with the arms crossed over the chest, six sit-to-stand with extra load, one unilateral sit-to-stand, and one unilateral sit-to-stand with extra load. The 15 patients completing two sets at home were distributed in STAND as follows: three sit-to-stand using the arm rests when standing up and sitting down, one sit-to-stand sit-to-stand with extra load, one unilateral sit-to-stand, and two unilateral sit-to-stand with extra load (Tabel 3). The mean Borg score when performing the highest level possible was $14.2( \pm 1.9)$ on admission and $14.1( \pm 1.6)$ at follow-up. 
307

308

309

310

311

312

313

314

315

316

317

318

319

320

321

322

323

324

325

326

327

328

329

330

331

332

333

334

335

336

337

\section{Indicators offloor/ceiling effect}

Two patients were at the lowest level of STAND at the hospital (knee-extensions with three and six kg, respectively). For both patients, a possibility of further regression was possible by using less weight (they both performed the exercise at level 3 at home). One patient was at the highest level of STAND at the hospital and two were at the highest level at home (unilateral sit-to-stand with six $\mathrm{kg}$ and four $\mathrm{kg}$, respectively) - for both patients a possibility of further progression was possible by adding more weight.

\section{Pain}

Four patients and two patients, respectively, reported an increase in pain after the DEMMI test at the hospital and at home. None of these patients reported any pain before the exercise.

Four patients reported light to moderate pain in the shoulder, leg and chest, respectively, before performing the exercise at the hospital. The pain remained unchanged during and after the exercise for three of the patients and one patient reported no pain after ended exercise. Three patients reported light leg pain during the exercise but no pain before and after the exercise. Four patients reported light to moderate pain in the shoulder, back, leg and head, respectively, before performing the exercise at home. The pain remained unchanged during and after the exercise for three of the patients and one patient reported less pain after ended exercise. Two patients reported light back pain during the exercise but no pain before and after the exercise.

\section{Mobility and cognition}

As shown in Figure 3 those that scored higher on the DEMMI performed the exercise at the most challenging levels of STAND (on admission, $\beta=0.10(\mathrm{CI}: 0.07 ; 0.13$ ), $\mathrm{P}<0.0001$; at home, $\beta=0.07$ (CI:0.03;0.12), $\mathrm{P}=0.004)$, whereas the level of STAND did not depend significantly on OMC (on admission: 0.07(-0.12;0.26), $\mathrm{P}=0.45$; at home: $-0.01(-0.42 ; 0.41), \mathrm{P}=0.96)$.

\section{Discussion}

The major finding of our feasibility study was that our exercise model of progressive sit-tostands (STAND) was feasible among hospitalized older adults and demonstrated potential for being used in a future study appropriately powered to evaluate the effect of the exercise on mobility, physical activity, functional performance and independence in this population. 
338 Specifically, we found that more than $75 \%$ of the patients assessed during hospitalization and

339 shortly following discharge in their own home were able to perform the sit-to-stand exercise at a 340 given level of STAND reaching an intensity of 8-12 RM for 8-12 repetitions. No clustering of

341 patients at the highest or lowest level of STAND was seen, suggesting no ceiling or floor effect,

342 and for all patients assessed a possibility of either progression or regression was possible.

343 Finally, no adverse events were reported.

344

345 Consistent with this study, previous studies have found resistance training to be feasible in older 346 hospitalized patients (Siebens et al., 2000; Mallery et al., 2003). However, these studies have

347 used either low intensity exercises; due to a concern of potential risks of exercising older

348 hospitalized patients (Siebens et al., 2000); or exercises performed lying in bed (Mallery et al.,

349 2003). Our study shows that a performance-based, higher-intensity exercise is feasible both in

350 hospitalized older adults with high and low mobility (Macri et al., 2012) (a DEMMI score of 44-

351 80) and with and without mild cognitive impairment (Katzman et al., 1983) (an OMC score of

352 18-28). Moreover, we found a strong association between the level of STAND and DEMMI

353 which indicates that the achieved level of STAND reflected the mobility level of the patients.

354 Additionally, the level of STAND was not associated with cognition, which implies that STAND

355 can be used independent of cognitive level. It has previously been shown that high intensity

356 resistance training is superior to low intensity in frail older adults (Seynnes et al., 2004), which is

357 why STAND may be a good choice in older hospitalized adults. We were able to provide optimal

358 resistances with the exercise as more than $75 \%$ of the assessed patients were able to perform the

359 exercise with a loading of 8-12 RM for 8-12 repetitions for the intended number of sets. Of those

360 not able to reach the intended loading/number of sets two thirds stopped after 6-7 repetitions or

361 due to muscular fatigue. This may indicate that they were able to perform the exercise but

362 needed better adjustment of the load or needed better information regarding the management of

363 muscular fatigue when performing strength training. The mean Borg score when performing the

364 highest level possible was 14, corresponding to a 75\% effort (Avers \& Brown, 2009). Thus, this

365 subjectively perceived effort corresponds well with 8-12 RM (Kraemer \& Ratamess, 2004) and

366 indicates that the patients have exercised at the intended level. Also, no adverse events were

367 seen. Therefore, this mode of progressive exercise seems appropriate as a simple strength

368 training exercise in acutely admitted older medical patients. 


\section{Limitations and strengths}

371 A limitation of the study is that the assessed patients represent a select group of acutely admitted

372 older medical patients as $90 \%$ of the patients fulfilling the inclusion criteria were either excluded

$373(80 \%)$ or declined to participate $(10 \%)$. The proportion of patients consenting to participate,

374 however, is equal to (Mallery et al., 2003) or higher (Siebens et al., 2000; Brown et al., 2006)

375 than seen in previous exercise studies in older hospitalized adults, which underlines the difficulty

376 of including patients in the acute setting and limits the generalizability to acutely admitted older

377 patients equivalent to our sample. In addition, we consider our exclusion criteria reasonable as

378 the majority of those excluded either would probably not have been able to perform the exercise

379 with the intended quality (e.g. due to dementia or confusion; 32.5\%), or would not benefit from a

380 program including the exercise (e.g. due to being in cancer treatment or terminally ill; $7.5 \%$ ) or

381 had a very short hospital stay (discharged within the first 24 hours; 53\%). However, patients

382 excluded due to inability to rise from a chair might benefit from exercise based on the STAND

383 model (level 1) or other interventions based on less demanding exercises equivalent with the

384 ones used by Mallery and co-workers (Mallery et al., 2003). Another limitation of our study is

385 that the feasibility of STAND has only been tested for one session in each setting (hospital and

386 home) and therefore, we are not able to evaluate whether the patients can comply with the

387 exercise over time or whether STAND is sufficient in ensuring the right load over time, e.g. a

388 training period of 4 weeks. We do believe, though, that the model can be used for a longer

389 training period, as progression and regression was possible for all levels of the model and neither

390 floor nor ceiling effect was seen.

391

392 A major strength of our study is that the exercise, following STAND, is well-described, simple

393 and low in cost making it possible to implement both in an acute hospital ward as well as in the

394 patients' homes. A study by Sullivan et al. (Sullivan et al., 2001) in hospitalized frail elderly

395 showed that 10 weeks of resistance training consisting of three sets of eight leg presses in a leg

396 press chair increased strength and lowered sit-to-stand time. The sit-to-stand exercise (level 2-8

397 of STAND) corresponds well with the leg press exercise, requiring the use of similar muscle-

398 synergies. However, in the hospital and especially in the home setting weight-lifting equipment

399 like a leg press chair is not often available why it is promising that using a weight vest and the 
400 sit-to-stand exercise patients can be loaded to the same extend enabling low technology

401 resistance training both in the hospital and at home. Additionally, as expressed in several recent

402 reviews it is very important to use exercise programs that are detailed with regard to technique,

403 dosage and progression of the exercise. Our program complies with the recommendation (De

404 Morton, Keating \& Jeffs, 2009b; Liu \& Latham, 2009; Steib, Schoene \& Pfeifer, 2010; Kosse et

405 al., 2013; Giné-Garriga et al., 2014; Timmer, Unsworth \& Taylor, 2014; White et al., 2015).

406 Moreover, the inclusion of physiotherapist supervision ensures optimal dosage and technique and

407 may also enhance compliance. This design element was included to overcome challenges within

408 previous studies that used unsupervised training in the home setting (Siebens et al., 2000; Buhl et

409 al., 2015).

410

\section{Perspective}

412 We are now conducting a randomized controlled trial to test a cross-continuum strength training

413 intervention in older medical patients (NCT01964482). The goal of the trial is to investigate the

414 effect of a simple, supervised strength training program consisting of two lower-extremity

415 strength training exercises. The exercises are based on STAND and performed during

416 hospitalization and the first four weeks after discharge at home.

417

418 Conclusions

419 Based on our pre-defined criteria for feasibility we found that a simple progression model for

420 loaded sit-to-stands (STAND) was feasible in acutely admitted older medical patients (+65 yrs)

421 in the hospital- and home setting. Following the progression model, a strength-training intensity

422 of 8-12 RM for 8-12 repetitions was reached for two thirds of the assessed patients with no

423 indication of ceiling or floor effect for load, and no report of adverse events.

424

425

426 Acknowledgements

427 Thanks to the physiotherapists in the municipality of Copenhagen for helping in the development

428 of the model.

429 
430 References

431 Abbott JH. 2014. The distinction between randomized clinical trials (RCTs) and preliminary

432

434

435

436

437

438

439

440

441

442

443

444

445

446

447

448

449

450

451

452

453 feasibility and pilot studies: what they are and are not. The Journal of Orthopaedic and Sports Physical Therapy 44:555-558.

Abrahin O, Rodrigues RP, Nascimento VC, Da Silva-Grigoletto ME, Sousa EC, Marçal AC. 2014. Single- and multiple-set resistance training improves skeletal and respiratory muscle strength in elderly women. Clinical Interventions in Aging 9:1775-1782.

Alexander NB, Galecki AT, Grenier ML, Nyquist LV, Hofmeyer MR, Grunawalt JC, Medell JL, Fry-Welch D. 2001. Task-Specific Resistance Training to Improve the Ability of Activities of Daily Living-Impaired Older Adults to Rise from a Bed and from a Chair. Journal of the American Geriatrics Society 49:1418-1427.

Alley DE, Koster A, Mackey D, Cawthon P, Ferrucci L, Simonsick EM, Yu B, Hardy S, Goodpaster B, Sarkisian C, Houston DK, Kritchevsky SB, Cummings S, Lee J-S, Tylavsky FA, Newman A, Harris T. 2010. Hospitalization and change in body composition and strength in a population-based cohort of older persons. Journal of the American Geriatrics Society 58:2085-2091.

Arain M, Campbell MJ, Cooper CL, Lancaster GA. 2010. What is a pilot or feasibility study? A review of current practice and editorial policy. BMC medical research methodology $10: 67$.

Avers D, Brown M. 2009. White paper: Strength training for the older adult. Journal of Geriatric Physical Therapy (2001) 32:148-152, 158.

Bassey EJ, Fiatarone MA, O’Neill EF, Kelly M, Evans WJ, Lipsitz LA. 1992. Leg extensor power and functional performance in very old men and women. Clinical science (London, England: 1979) 82:321-327. 
454 Bean JF, Kiely DK, Herman S, Leveille SG, Mizer K, Frontera WR, Fielding RA. 2002. The

455

456

457

458

461

462

463

464

465

466

467

468

469

470

471

472

473

474

475

476 relationship between leg power and physical performance in mobility-limited older people. Journal of the American Geriatrics Society 50:461-467.

Bean JF, Herman S, Kiely DK, Frey IC, Leveille SG, Fielding RA, Frontera WR. 2004. Increased Velocity Exercise Specific to Task (InVEST) training: a pilot study exploring effects on leg power, balance, and mobility in community-dwelling older women. Journal of the American Geriatrics Society 52:799-804.

Bodilsen AC, Pedersen MM, Petersen J, Beyer N, Andersen O, Smith LL, Kehlet H, Bandholm T. 2013. Acute Hospitalization of the Older Patient: Changes in Muscle Strength and Functional Performance During Hospitalization and 30 Days After Discharge. American journal of physical medicine \& rehabilitation / Association of Academic Physiatrists.

Borg G. 1970. Perceived exertion as an indicator of somatic stress. Scandinavian journal of rehabilitation medicine 2:92-98.

Bowen DJ, Kreuter M, Spring B, Cofta-Woerpel L, Linnan L, Weiner D, Bakken S, Kaplan CP, Squiers L, Fabrizio C, Fernandez M. 2009. How We Design Feasibility Studies. American Journal of Preventive Medicine 36:452-457.

Boyd CM, Landefeld CS, Counsell SR, Palmer RM, Fortinsky RH, Kresevic D, Burant C, Covinsky KE. 2008. Recovery of Activities of Daily Living in Older Adults After Hospitalization for Acute Medical Illness. Journal of the American Geriatrics Society $56: 2171-2179$.

Brown CJ, Peel C, Bamman MM, Allman RM. 2006. Exercise program implementation proves not feasible during acute care hospitalization. Journal of rehabilitation research and development 43:939-946. 
477 Brown CJ, Friedkin RJ, Inouye SK. 2004. Prevalence and outcomes of low mobility in 478 hospitalized older patients. Journal of the American Geriatrics Society 52:1263-1270.

479 Buhl SF, Andersen AL, Andersen JR, Andersen O, Jensen J-EB, Rasmussen AML, Pedersen 480 MM, Damkjær L, Gilkes H, Petersen J. 2015. The effect of protein intake and resistance training on muscle mass in acutely ill old medical patients - A randomized controlled trial. Clinical Nutrition (Edinburgh, Scotland).

483 De Buyser SL, Petrovic M, Taes YE, Vetrano DL, Corsonello A, Volpato S, Onder G. 2014. Functional Changes during Hospital Stay in Older Patients Admitted to an Acute Care Ward: A Multicenter Observational Study. PloS one 9:e96398.

Chandler JM, Duncan PW, Kochersberger G, Studenski S. 1998. Is lower extremity strength gain associated with improvement in physical performance and disability in frail, communitydwelling elders? Archives of physical medicine and rehabilitation 79:24-30.

Coker RH, Hays NP, Williams RH, Wolfe RR, Evans WJ. 2014. Bed Rest Promotes Reductions in Walking Speed, Functional Parameters, and Aerobic Fitness in Older, Healthy Adults. The Journals of Gerontology. Series A, Biological Sciences and Medical Sciences. Improved functional ability and independence in activities of daily living for older adults at high risk of hospital readmission: a randomized controlled trial. Journal of Evaluation in Clinical Practice 18:128-134.

Covinsky KE, Palmer RM, Fortinsky RH, Counsell SR, Stewart AL, Kresevic D, Burant CJ, Landefeld CS. 2003. Loss of independence in activities of daily living in older adults hospitalized with medical illnesses: increased vulnerability with age. Journal of the American Geriatrics Society 51:451-458. 
500 Von Elm E, Altman DG, Egger M, Pocock SJ, Gøtzsche PC, Vandenbroucke JP, for the

501 STROBE Initiative. 2014. The Strengthening the Reporting of Observational Studies in

502 Epidemiology (STROBE) Statement: Guidelines for reporting observational studies.

$503 \quad$ International Journal of Surgery (London, England).

504 Foss NB, Kristensen MT, Kehlet H. 2006. Prediction of postoperative morbidity, mortality and rehabilitation in hip fracture patients: the cumulated ambulation score. Clinical Rehabilitation 20:701-708.

507 Fried TR, Tinetti ME, Iannone L, O’Leary JR, Towle V, Van Ness PH. 2011. Health outcome

508 prioritization as a tool for decision making among older persons with multiple chronic conditions. Archives of Internal Medicine 171:1854-1856.

510 Giné-Garriga M, Roqué-Fíguls M, Coll-Planas L, Sitjà-Rabert M, Salvà A. 2014. Physical exercise interventions for improving performance-based measures of physical function in community-dwelling, frail older adults: a systematic review and meta-analysis. Archives of physical medicine and rehabilitation 95:753-769.e3.

514 Hoffmann TC, Glasziou PP, Boutron I, Milne R, Perera R, Moher D, Altman DG, Barbour V,

515 Macdonald H, Johnston M, Lamb SE, Dixon-Woods M, McCulloch P, Wyatt JC, Chan

516 A-W, Michie S. 2014. Better reporting of interventions: template for intervention

517 description and replication (TIDieR) checklist and guide. BMJ (Clinical research ed.)

$518 \quad 348: g 1687$.

519 Hvid L, Aagaard P, Justesen L, Bayer ML, Andersen JL, Ørtenblad N, Kjaer M, Suetta C. 2010.

520 Effects of aging on muscle mechanical function and muscle fiber morphology during 521 short-term immobilization and subsequent retraining. Journal of applied physiology

522 (Bethesda, Md.: 1985) 109:1628-1634. 
523 Hvid LG, Suetta C, Nielsen JH, Jensen MM, Frandsen U, Ørtenblad N, Kjaer M, Aagaard P.

524 2014. Aging impairs the recovery in mechanical muscle function following 4 days of 525 disuse. Experimental Gerontology 52:1-8.

526 Katzman R, Brown T, Fuld P, Peck A, Schechter R, Schimmel H. 1983. Validation of a short Orientation-Memory-Concentration Test of cognitive impairment. The American Journal of Psychiatry 140:734-739.

Kondrup J. 2003. ESPEN Guidelines for Nutrition Screening 2002. Clinical Nutrition 22:415421.

531

Koopman R, van Loon LJC. 2009. Aging, exercise, and muscle protein metabolism. Journal of Applied Physiology (Bethesda, Md.: 1985) 106:2040-2048.

Kortebein P, Ferrando A, Lombeida J, Wolfe R, Evans WJ. 2007. Effect of 10 days of bed rest on skeletal muscle in healthy older adults. JAMA: the journal of the American Medical Association 297:1772-1774.

Kortebein P. 2009. Rehabilitation for Hospital-Associated Deconditioning. American Journal of Physical Medicine \& Rehabilitation 88:66-77.

Kosse NM, Dutmer AL, Dasenbrock L, Bauer JM, Lamoth CJC. 2013. Effectiveness and feasibility of early physical rehabilitation programs for geriatric hospitalized patients: a systematic review. BMC geriatrics 13:107.

Kraemer WJ, Adams K, Cafarelli E, Dudley GA, Dooly C, Feigenbaum MS, Fleck SJ, Franklin B, Fry AC, Hoffman JR, others. 2002. American College of Sports Medicine position stand. Progression models in resistance training for healthy adults. Medicine and science in sports and exercise 34:364. 
545 Kraemer WJ, Ratamess NA. 2004. Fundamentals of Resistance Training: Progression and $546 \quad$ Exercise Prescription. Medicine \& Science in Sports \& Exercise 36:674-688.

547 Liu CJ, Latham NK. 2009. Progressive resistance strength training for improving physical 548 function in older adults. Cochrane Database Syst Rev 3.

549 Macri EM, Lewis JA, Khan KM, Ashe MC, de Morton NA. 2012. The de Morton Mobility Index: Normative Data for a Clinically Useful Mobility Instrument. Journal of Aging Research 2012.

552

553

554

555

556

557 558

Mallery LH, MacDonald EA, Hubley-Kozey CL, Earl ME, Rockwood K, MacKnight C. 2003. The feasibility of performing resistance exercise with acutely ill hospitalized older adults. BMC geriatrics 3:3.

Melzack R. 1975. The McGill Pain Questionnaire: major properties and scoring methods. Pain $1: 277-299$.

De Morton NA, Davidson M, Keating JL. 2008. The de Morton Mobility Index (DEMMI): An essential health index for an ageing world. Health and Quality of Life Outcomes 6:63.

De Morton N, Keating JL, Jeffs K. 1996. Exercise for acutely hospitalised older medical patients. In: Cochrane Database of Systematic Reviews. John Wiley \& Sons, Ltd,.

De Morton NA, Keating JL, Jeffs K. 2009a. Exercise for acutely hospitalised older medical patients. Cochrane Database Syst Rev 1.

De Morton NA, Keating JL, Jeffs K. 2009b. Exercise for acutely hospitalised older medical patients. Cochrane Database Syst Rev 1.

Mudge AM, O'Rourke P, Denaro CP. 2010. Timing and risk factors for functional changes associated with medical hospitalization in older patients. The journals of gerontology. Series A, Biological sciences and medical sciences 65:866-872. 
568 Nicola F, Catherine S. 2011. Dose-response relationship of resistance training in older adults: a 569 meta-analysis. British Journal of Sports Medicine 45:233-234.

570 Nolan J, Thomas S. 2008. Targeted individual exercise programmes for older medical patients

571

572

573

574

575

576

577

578

579

580

581

582

583

584

585

586

587

588

589

are feasible, and changes hospital and patient outcomes: a service improvement project. BMC Health Services Research 8:250.

Oakland HT, Farber JI. 2014. The effect of short, unplanned hospitalizations on older adult functional status. Journal of the American Geriatrics Society 62:788-789.

Parker MJ, Palmer CR. 1993. A new mobility score for predicting mortality after hip fracture. The Journal of bone and joint surgery. British volume 75:797-798.

Pedersen MM, Bodilsen AC, Petersen J, Beyer N, Andersen O, Lawson-Smith L, Kehlet H, Bandholm T. 2012. Twenty-Four-Hour Mobility During Acute Hospitalization in Older Medical Patients. The journals of gerontology. Series A, Biological sciences and medical sciences.

Rabin R, de Charro F. 2001. EQ-5D: a measure of health status from the EuroQol Group. Annals of medicine 33:337-343.

Raymond MJ, Bramley-Tzerefos RE, Jeffs KJ, Winter A, Holland AE. 2013. Systematic Review of High-Intensity Progressive Resistance Strength Training of the Lower Limb Compared With Other Intensities of Strength Training in Older Adults. Archives of Physical Medicine and Rehabilitation 94:1458-1472.

Row BS, Knutzen KM, Skogsberg NJ. 2012. Regulating explosive resistance training intensity using the rating of perceived exertion. Journal of Strength and Conditioning Research / National Strength \& Conditioning Association 26:664-671. 
590 Saltin B, Grimby G. 1968. Physiological analysis of middle-aged and old former athletes.

591 Comparison with still active athletes of the same ages. Circulation 38:1104-1115.

592 Schnohr P, Scharling H, Jensen JS. 2003. Changes in leisure-time physical activity and risk of

593 death: an observational study of 7,000 men and women. American Journal of

$594 \quad$ Epidemiology 158:639-644.

595 Seynnes O, Fiatarone Singh MA, Hue O, Pras P, Legros P, Bernard PL. 2004. Physiological and 596 functional responses to low-moderate versus high-intensity progressive resistance training in frail elders. The journals of gerontology. Series A, Biological sciences and

Siebens H, Aronow H, Edwards D, Ghasemi Z. 2000. A randomized controlled trial of exercise to improve outcomes of acute hospitalization in older adults. Journal of the American

Steib S, Schoene D, Pfeifer K. 2010. Dose-Response Relationship of Resistance Training in Geriatrics Society 48:1545-1552.

604 605 606 Older Adults. Medicine \& Science in Sports \& Exercise 42:902-914.

Stewart VH, Saunders DH, Greig CA. 2014. Responsiveness of muscle size and strength to physical training in very elderly people: A systematic review. Scandinavian journal of medicine \& science in sports 24:e1-e10.

Suetta C, Magnusson SP, Beyer N, Kjaer M. 2007. Effect of strength training on muscle function in elderly hospitalized patients. Scandinavian Journal of Medicine \& Science in Sports $17: 464-472$.

Sullivan DH, Wall PT, Bariola JR, Bopp MM, Frost YM. 2001. Progressive resistance muscle strength training of hospitalized frail elderly. American journal of physical medicine \& rehabilitation / Association of Academic Physiatrists 80:503-509. 
613 Tan B. 1999. Manipulating Resistance Training Program Variables to Optimize Maximum

614 Strength in Men: A Review. Journal of Strength and Conditioning Research 13:289-304.

615 Tibaek S, Andersen CW, Pedersen SF, Rudolf KS. 2013. Does progressive resistance strength

616 training as additional training have any measured effect on functional outcomes in older

617 hospitalized patients? A single-blinded randomized controlled trial. Clinical

$618 \quad$ Rehabilitation:0269215513501524.

619 Timmer AJ, Unsworth CA, Taylor NF. 2014. Rehabilitation interventions with deconditioned

620 older adults following an acute hospital admission: a systematic review. Clinical

621 rehabilitation.

622 Villumsen M, Jorgensen MG, Andreasen J, Rathleff MS, Mølgaard CM. 2014. Very Low Levels

623 of Physical Activity in Older Patients During Hospitalization at an Acute Geriatric Ward

624 - A Prospective Cohort Study. Journal of Aging and Physical Activity.

625 Wade DT, Vergis E. 1999. The Short Orientation-Memory-Concentration Test: a study of its

626 reliability and validity. Clinical Rehabilitation 13:164-170.

627 Wall BT, Dirks ML, van Loon LJC. 2013. Skeletal muscle atrophy during short-term disuse:

628 Implications for age-related sarcopenia. Ageing research reviews 12:898-906.

629 White NT, Delitto A, Manal TJ, Miller S. 2015. The American Physical Therapy Association's

630 top five choosing wisely recommendations. Physical Therapy 95:9-24.

631 Zisberg A, Shadmi E, Gur-Yaish N, Tonkikh O, Sinoff G. 2015. Hospital-associated functional

632 decline: the role of hospitalization processes beyond individual risk factors. Journal of

633 the American Geriatrics Society 63:55-62.

634

635 
636 Table 1. Patient characteristics on admission.

\begin{tabular}{|c|c|c|}
\hline & $\mathrm{N}$ & \\
\hline Age; mean (SD) & 24 & $77 \pm 7$ \\
\hline Gender, female; n (\%) & 24 & $12(50 \%)$ \\
\hline Living alone, yes; $\mathrm{n}(\%)$ & 24 & $13(54 \%)$ \\
\hline Use of gait devices, yes; $\mathrm{n}(\%)$ & 24 & $9(37.5 \%)$ \\
\hline $\begin{array}{l}\text { Reason for admission; } \mathrm{n}(\%) \\
\text { Pneumonia } \\
\text { COPD exacerbation } \\
\text { Dyspnea } \\
\text { Urinary tract infection } \\
\text { Gastroenteritis } \\
\text { Pulmonary embolism } \\
\text { Atrial fibrillation } \\
\text { Anemia }\end{array}$ & 24 & $\begin{array}{l}10(41.7 \%) \\
2(8.3 \%) \\
1(4.2 \%) \\
3(12.5 \%) \\
1(4.2 \%) \\
2(8.3 \%) \\
3(12.5 \%) \\
2(8.3 \%)\end{array}$ \\
\hline $\begin{array}{l}\text { Physical activity level (PA); n (\%) } \\
\text { Low PA } \\
\text { Moderate PA } \\
\text { High PA } \\
\end{array}$ & 23 & $\begin{array}{l}5(21.7 \%) \\
5(21.7 \%) \\
13(56.6 \%)\end{array}$ \\
\hline Comorbidities; $\mathrm{n}$ (IQR) & 24 & $5(3.5 ; 5.5)$ \\
\hline Medications; $\mathrm{n}$ (IQR) & 24 & $6(2.5 ; 7.5)$ \\
\hline Length of stay; median (IQR) & 24 & $4.5(3 ; 7)$ \\
\hline Follow-up - number of days after discharge; median (IQR) & 19 & $9(6 ; 13)$ \\
\hline $\begin{array}{l}\text { Nutritional Risk Screening } \\
\text { At risk; n }(\%)\end{array}$ & 24 & $19(79.2 \%)$ \\
\hline OMC; median (IQR)/n(\%) & 24 & $26(22 ; 28)$ \\
\hline CAS; median (IQR) & 24 & $6(6 ; 6)$ \\
\hline NMS, 14 days prior to admission; median (IQR) & 24 & $9(5.5 ; 9)$ \\
\hline NMS at admission; median (IQR) & 24 & $3(2 ; 9)$ \\
\hline DEMMI; mean (SD) & 23 & $66.1+/-15.18$ \\
\hline
\end{tabular}

OMC: The Short Orientation-Memory-Concentration test; CAS: The Cumulated Ambulation Score;

NMS: The New Mobility Score; DEMMI: The de Morton Mobility Index

637

638 
Table 2. Performance measures on admission and at home.

\begin{tabular}{|l|l|l|l|l|l|}
\hline Performance measure & $\mathrm{N}$ & Admission & $\mathrm{N}$ & Home-visit & P-value \\
\hline CAS; median (IQR) & 24 & $6(6 ; 6)$ & 20 & $6(6 ; 6)$ & NA* \\
\hline NMS admission; median (IQR) & 24 & $3(2 ; 9)$ & 20 & $6.5(3 ; 9)$ & 0.13 \\
\hline DEMMI; mean (SD) & 23 & $66.1(15.18)$ & 19 & $70.6(14.7)$ & 0.12 \\
\hline EQ-VAS; mean (SD) & 24 & $56.6(24.3)$ & 20 & $67.4(23.8)$ & 0.01 \\
\hline
\end{tabular}

*no participants changed in CAS.

639

640 
641 Table 3. Overview over the 8 levels of the STAND model and the distribution of patients on the 6428 levels according to the highest level performed in the hospital and at home, respectively.

\begin{tabular}{|c|c|c|c|c|}
\hline $\begin{array}{l}\text { Level in } \\
\text { STAND }\end{array}$ & Description of level & Illustration & $\begin{array}{l}\text { In hospital } \\
\text { (n) }\end{array}$ & At home (n) \\
\hline 1 & $\begin{array}{c}\text { Seated knee extensions } \\
\text { with or without added load, } \\
\text { e.g. weight cuffs. }\end{array}$ & & 2 & 0 \\
\hline 2 & $\begin{array}{l}\text { STS with armrest support } \\
\text { and support from another } \\
\text { person allowed; } \\
\text { own body weight. }\end{array}$ & & 0 & 0 \\
\hline 3 & $\begin{array}{l}\text { STS with armrest support } \\
\text { in eccentric and concentric } \\
\text { phase allowed; } \\
\text { own body weight. }\end{array}$ & & 2 & 3 \\
\hline 4 & $\begin{array}{l}\text { STS with armrest support } \\
\text { in concentric phase } \\
\text { allowed; } \\
\text { own body weight. }\end{array}$ & & 2 & 1 \\
\hline $\begin{array}{c}5 \\
\text { Starting point }\end{array}$ & $\begin{array}{l}\text { STS without support; } \\
\text { own body weight. }\end{array}$ & & 6 & 4 \\
\hline 6 & $\begin{array}{l}\text { STS with added load; } \\
\text { e.g. weight vest. }\end{array}$ & & 6 & 4 \\
\hline 7 & $\begin{array}{c}\text { Unilateral STS with } \\
\text { balance support allowed; } \\
\text { own body weight }\end{array}$ & & 1 & 1 \\
\hline 8 & $\begin{array}{l}\text { Unilateral STS with } \\
\text { balance support allowed } \\
\text { and with added load; } \\
\text { e.g.weight vest. }\end{array}$ & 8 & 1 & 2 \\
\hline
\end{tabular}


Figure 1. Progression model for loaded sit-to-stand exercise (STAND)

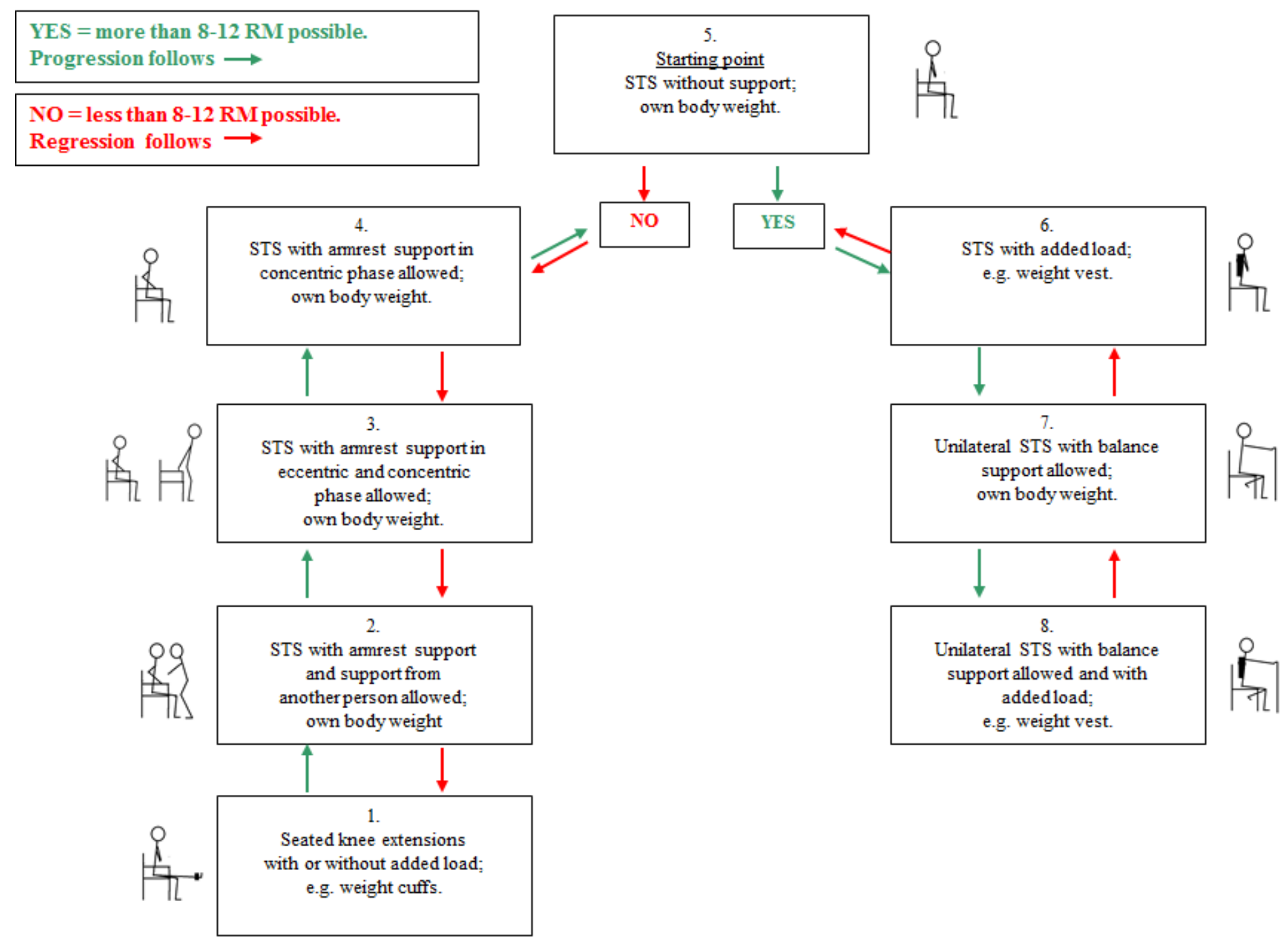




\section{Figure 1 bis. Description of model-procedure \\ Preparation}

Seated on a standard chair with armrests, and a seat height of approximately $45 \mathrm{~cm}$, the individual should perform 5 unloaded knee extensions for each limb as a warm-up.

\section{Procedure}

- Perform all exercises at a moderate velocity with both the concentric (raising) and the eccentric (lowering) component being performed over 2 seconds, separated by a 1-second quasi-isometric pause after the concentric and eccentric phases, respectively.

- Perform as many repetitions as possible maintaining the same pace to ensure training to muscular fatigue.

- If muscular fatigue is reached within 8-12 repetitions, stay at the same level.

- If muscular fatigue is reached before 8 repetitions, perform the exercise at a lower level.

- If muscular fatigue is reached after more than 12 repetitions, perform the exercise at a higher level.

- Aim at 3 sets of 8-12 repetitions to muscular fatigue ( $3 \times 8-12 \mathrm{RM})$.

- Allow minimal extra support after 6 non-compensatory repetitions to attain muscular fatigue - if a proper technique is maintained.

- Allow increased speed in the last two repetitions if necessary to ensure training at the highest possible level.

- Adjust loads/levels on a set-by-set basis.

- Ensure a 1-minute pause between sets.

\section{Levels - the starting point is level 5:}

All levels are started from a seated position.

Level 1: Attach an appropriate weight cuff $(\geq 0.5 \mathrm{~kg})$ around the ankle. Fully extend the knee and bend it reaching $90^{\circ}$ flexion.

Level 2: From a seated position, rise to a fully extended position and sit down using the armrests as support and with additional support from the physiotherapist.

Level 3: From a seated position, rise to a fully extended position and sit down using the armrests as support.

Level 4: From a seated position, rise to a fully extended position using the armrests as support. Sit down with the arms crossed over the chest.

Level 5: From a seated position with arm crossed over the chest, rise to a fully extended position and sit down.

Level 6: From a seated position with arm crossed over the chest and wearing a weight vest (1-30 $\mathrm{kg})$, rise to a fully extended position and sit down.

Level 7: From a seated position (hands on chair in front of you for balance support), rise to a fully extended position on one leg and sit down (shift legs after each set, aiming at 3 set per leg)

Level 8: From a seated position wearing a weight vest (1-30 kg) (hands on chair in front of you for balance support), rise to a fully extended position on one leg and sit down (shift legs after each set, aiming at 3 set per leg) 


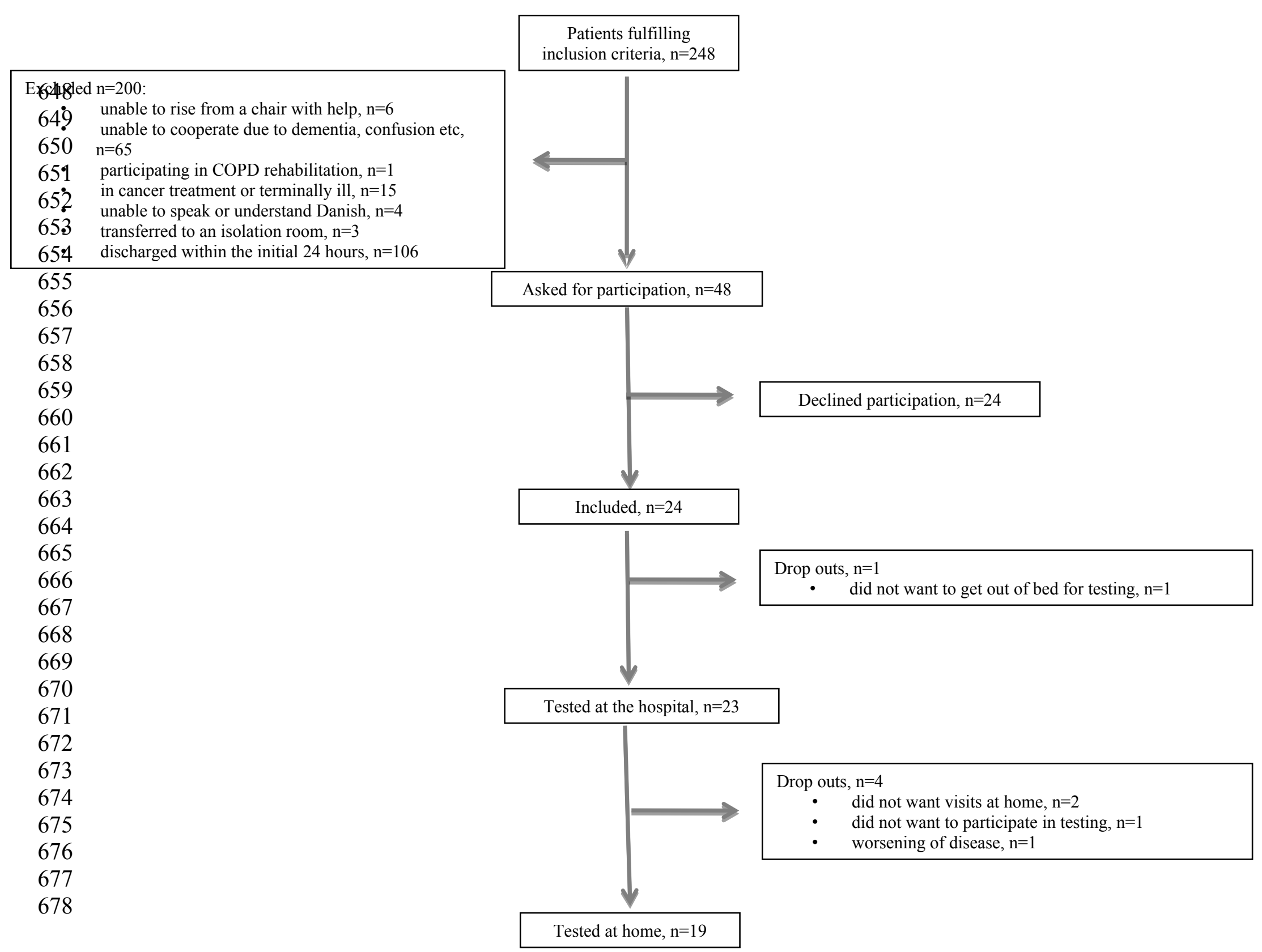

Figure 2 Flowchart 
Figure 3. The association between DEMMI score (A) and OMC score (B), respectively, and performed level of STAND on admission and at home.

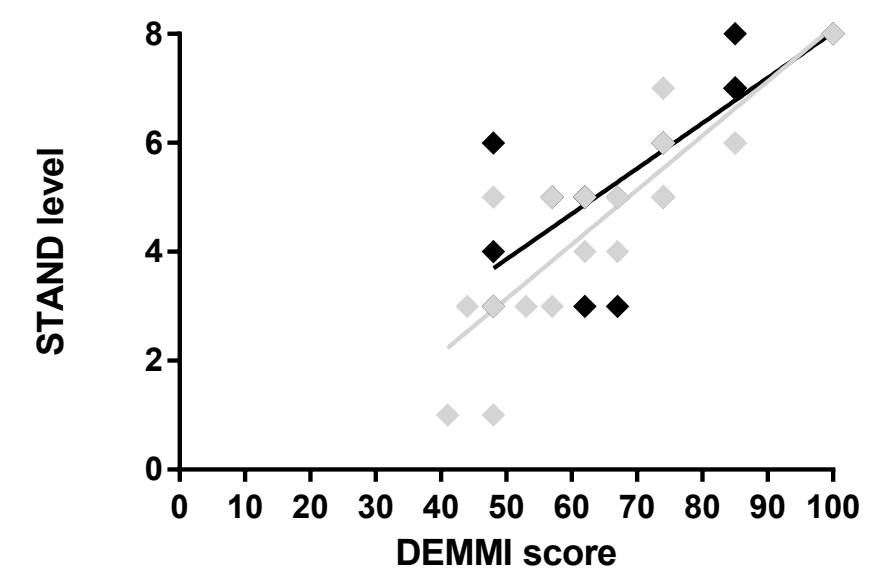

$\sim$ Admission
At home

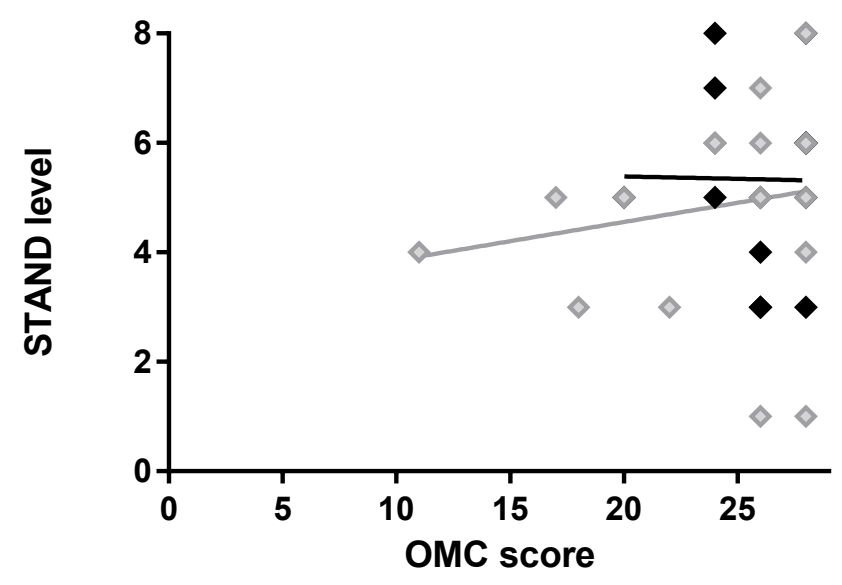

$\leadsto$ Admission $\quad \rightarrow$ At home

DEMMI score: score on the de Morton Mobility Index (0-100). The higher the score the better mobility.

OMC score: score on the Short Orientation-Memory-Concentration test (0-28). The higher the score the better cognition.

STAND level: 1 indicates lowest level of the model (seated knee-extensions) and 8 indicates highest level of the model (unilateral sit-

to-stand with added load). 